\title{
Grain size effect on the Néel temperature and magnetic properties of nanocrystalline $\mathrm{NiFe}_{2} \mathrm{O}_{4}$ spinel
}

\author{
C.N. Chinnasamy ${ }^{\mathrm{a}, 1}$, A. Narayanasamy ${ }^{\mathrm{a}, *}$, N. Ponpandian ${ }^{\mathrm{a}}$, R. Justin Joseyphus ${ }^{\mathrm{a}}$, \\ B. Jeyadevan ${ }^{\mathrm{b}}, \mathrm{K}$. Tohji ${ }^{\mathrm{b}}, \mathrm{K}$. Chattopadhyay ${ }^{\mathrm{c}}$ \\ ${ }^{a}$ Materials Science Centre, Department of Nuclear Physics, University of Madras, Guindy Campus, Chennai 600 025, India \\ ${ }^{\mathrm{b}}$ Department of Geoscience and Technology, Tohoku University Aramaki, Aoba-ku, Sendai 980-8579, Japan \\ ${ }^{\mathrm{c}}$ Department of Metallurgy, Indian Institute of Science, Bangalore 560 012, India
}

Received 26 June 2001

\begin{abstract}
Nanocrystalline $\mathrm{NiFe}_{2} \mathrm{O}_{4}$ spinel ferrites with various grain sizes have been synthesized by ball milling the bulk $\mathrm{NiFe}_{2} \mathrm{O}_{4}$. The average grain sizes were estimated from the X-ray line broadening of the (3 111$)$ reflection. The Néel temperatures of $\mathrm{NiFe}_{2} \mathrm{O}_{4}$ for various grain sizes were determined by magneto thermogravimetric method. The magnetic behaviour has been explained by combining the effects of changes in cation distribution on milling and finite size scaling. The shift in $B-H$ loops has been correlated to the surface spin effects. The high coercivities observed here may be due to high anisotropies of the milled samples. The Hopkinson peak observed just below the Néel temperature has been explained by the mathematical formalism given by the Stoner Wohlfarth model. (C) 2002 Elsevier Science B.V. All rights reserved.
\end{abstract}

PACS: 75.50.Tt; 75.50.Gg; 75.60.-d

Keywords: Néel temperature; Ferrimagnet; Hopkinson effect; Fine particle

\section{Introduction}

Since the last decade quite new and interesting magnetic properties have been reported for nanocrystalline spinel ferrites. The observation of large magnetic moments [1-10] and ferromagnetic or ferrimagnetic ordering [9-11] on grain size reduction to a few nanometer in the well-known low

\footnotetext{
*Corresponding author.

E-mail address: ansuom@yahoo.co.in (A. Narayanasamy).

${ }^{1}$ Department of Geoscience and Technology, Tohoku University Aramaki, Aoba-ku, Sendai 980-8579, Japan.
}

temperature antiferromagnetic spinels $\mathrm{ZnFe}_{2} \mathrm{O}_{4}$ and $\mathrm{CdFe}_{2} \mathrm{O}_{4}$ has been found to be quite exciting. The controversy with respect to the type of magnetic ordering namely, whether it is ferromagnetic or ferrimagnetic ordering is yet to be resolved. The role played by the method of synthesis of these materials on the magnetic properties cannot be ignored as materials from different laboratories are found to exhibit different magnetic properties [1-11]. The EXAFS study by Jeyadevan et al. [12] has shown that $\mathrm{ZnFe}_{2} \mathrm{O}_{4}$ undergoes a change in cation distribution from the normal spinel structure in the bulk form to mixed 
spinel structure in the nanocrystalline form. Nanocrystalline $\mathrm{NiFe}_{2} \mathrm{O}_{4}$ spinel is another such system which has been extensively studied by Mössbauer and magnetization techniques $[13,14]$. Recently, we have shown that nanocrystalline $\mathrm{NiFe}_{2} \mathrm{O}_{4}$ exhibits mixed spinel structure whereas in the bulk form it is a well-known inverse spinel with all $\mathrm{Ni}^{2+}$ ions on the $\mathrm{B}$-sites and $\mathrm{Fe}^{3+}$ ions equally distributed between A- and B- sites [15]. Our study has shown that the ball milled $\mathrm{NiFe}_{2} \mathrm{O}_{4}$ with a grain size of $60 \mathrm{~nm}$ is a mixed spinel with about four percent $\mathrm{Ni}^{2+}$ ions occupying the Asites. Also, the magnetization of $\mathrm{NiFe}_{2} \mathrm{O}_{4}$ spinel with grain size of $60 \mathrm{~nm}$ has been enhanced by $8 \%$ from its bulk value.

Chen et al. [16] have reported changes in cation distribution and a decrease in Néel temperature in $\mathrm{MnFe}_{2} \mathrm{O}_{4}$ as grain size is decreased. The decrease in Néel temperature has been found to be consistent with the finite size scaling. To our knowledge, this is the only spinel ferrite for which the grain size dependent Néel temperature has been reported. In the present study, we report the effect of grain size on Néel temperature in the case of $\mathrm{NiFe}_{2} \mathrm{O}_{4}$ spinel and also its effect on the magnetic properties such as saturation magnetization, coercivity and $B-H$ loop-shifts, etc. The coercivity reported in this paper is found to be the highest of all the values reported so far for the ultrafine particles of $\mathrm{NiFe}_{2} \mathrm{O}_{4}$.

\section{Experimental}

Polycrystalline nickel ferrite was synthesized by the conventional ceramic method and samples of various grain sizes were obtained by high energy ball milling in a planetary ball mill (Fritsch Pulverisette, P7) as reported earlier [15]. The formation of spinel phase was confirmed from X-ray powder diffraction measurements. Magnetic force measurements were carried out as a function of temperature with a thermogravimetric analyser (TGA, Perkin-Elmer series 7) using a small permanent magnet to determine the Néel temperature for various grain sizes. The samples were heated at a rate of $10^{\circ} \mathrm{C} / \mathrm{min}$. Magnetization measurements were performed with a Vibrating
Sample Magnetometer from 4 to $300 \mathrm{~K}$ in a magnetic field of $1 \mathrm{~T}$. The zero field-cooled (ZFC) magnetization data were obtained by cooling the sample to $4 \mathrm{~K}$ in zero field and then recording the $B-H$ loops in $1 \mathrm{~T}$ field at the interval of $10 \mathrm{~K}$ till $300 \mathrm{~K}$. The field-cooled (FC) magnetization data were obtained from the $B-H$ loops measured at $10 \mathrm{~K}$ interval while cooling the sample from 300 to $4 \mathrm{~K}$ in the presence of $1 \mathrm{~T}$ field.

\section{Results and discussion}

\subsection{Grain size, root mean square (r.m.s.) strain and lattice parameter}

The average grain size was determined from the line width of the reflection (3 1 1) in XRD patterns using Scherrer formula. The variation of average grain size with milling time is shown in Fig. 1. The grain size decreases with milling time and reaches $10 \mathrm{~nm}$ for the $30 \mathrm{~h}$ milled sample. The r.m.s. strain was calculated by using the relation [17]

$\beta=2 \eta\left\langle\mathrm{e}^{2}\right\rangle^{1 / 2} \tan \theta$,

where $\beta$ is the full-width at half-maximum of the X-ray Bragg peak and $\eta$ is the coefficient which depends on the distribution of strains, $\left\langle\mathrm{e}^{2}\right\rangle^{1 / 2}$ is

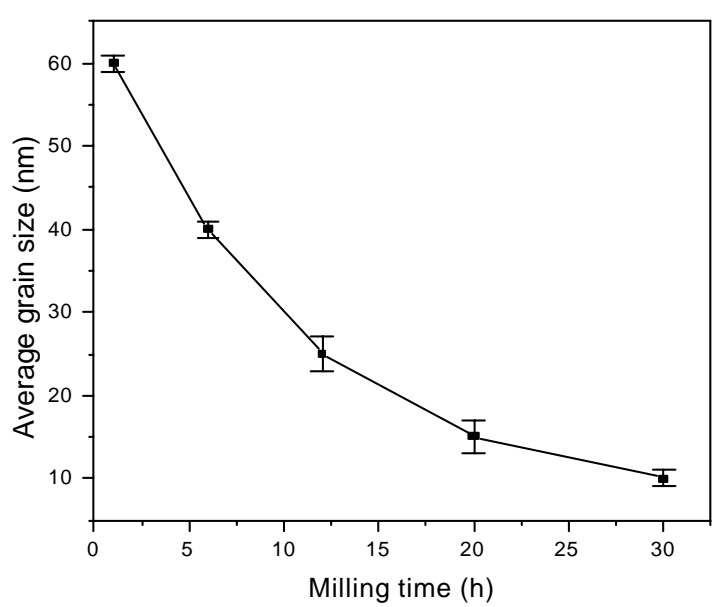

Fig. 1. Variation of average grain size of nanostructured $\mathrm{NiFe}_{2} \mathrm{O}_{4}$ with milling time. The continuous line is guide to the eye. 
the r.m.s. strain and $\theta$ is the diffraction angle and the values are plotted in Fig. 2 as a function of milling time. The increase in r.m.s. strain with milling is due to the increasing defect density. The lattice parameter as seen from Fig. 3 is higher for the milled samples than for the as-prepared one. This is in agreement with the findings of Sato et al. [18]. The increase in lattice parameter could be due to the deviation in cation distribution from the inverse spinel structure of the milled $\mathrm{NiFe}_{2} \mathrm{O}_{4}$ as confirmed from our EXAFS and in-field Mössbauer measurements [15].

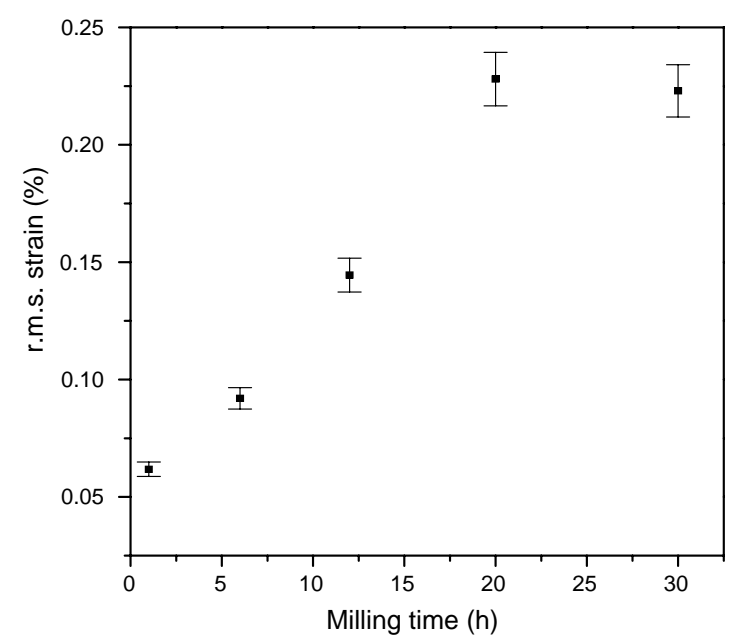

Fig. 2. The r.m.s. strain versus milling time for $\mathrm{NiFe}_{2} \mathrm{O}_{4}$.

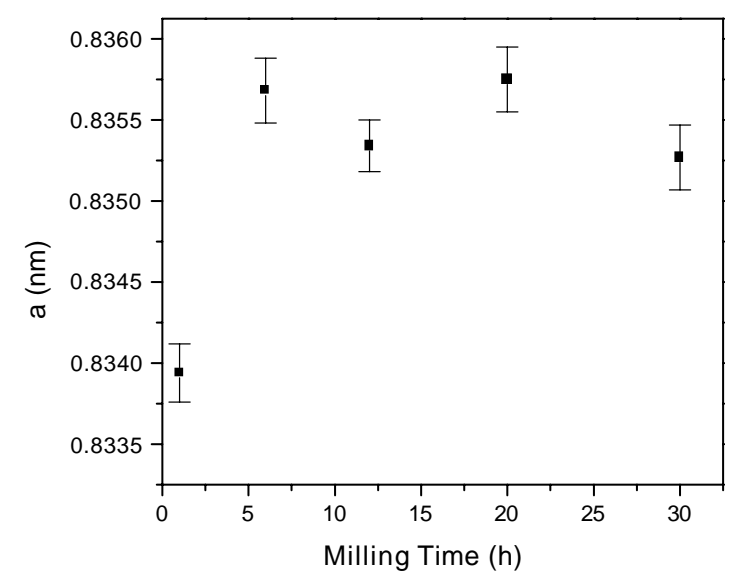

Fig. 3. The lattice parameter of the nanostructured $\mathrm{NiFe}_{2} \mathrm{O}_{4}$ as a function of milling time.

\subsection{Néel temperature versus grain size}

The Néel temperatures estimated from the thermogravimetric measurements for the $\mathrm{NiFe}_{2} \mathrm{O}_{4}$ samples with various grain sizes are shown in Fig. 4. As seen from the figure, the values of Néel temperatures of the milled samples are found to be greater than that of the reported value of $858 \mathrm{~K}$ for the bulk sample [19]. It increased with milling upto $6 \mathrm{~h}$ and thereafter it decreased with further milling. The decrease in the value of Néel temperature with grain size reduction in the case of $\mathrm{MnFe}_{2} \mathrm{O}_{4}$ has been explained on the basis of the finite size effects [16]. Similarly, the decrease in $T_{\mathrm{N}}$ with grain size at higher milling times for $\mathrm{NiFe}_{2} \mathrm{O}_{4}$ could be explained on the basis of the finite size scaling theory as seen from Fig. 5. According to this theory, the shift in the Néel temperature from that of the bulk is given by

$$
\frac{T_{\mathrm{N}}(d)-T_{\mathrm{N}}(\text { bulk })}{T_{\mathrm{N}}(\text { bulk })}=\left(\frac{d}{d_{0}}\right)^{-1 / v},
$$

where $T_{\mathrm{N}}(d)$ is the Néel temperature for a given particle size $d, T_{\mathrm{N}}$ (bulk) is the Néel temperature of the bulk sample, $d_{0}$ is a constant and $v$ is the critical exponent of the correlation lengths. The straight line in Fig. 5 is obtained as a result of fitting the experimental data using Eq. (2). The parameters obtained from the best fit were

$$
T_{\mathrm{N}}(\text { bulk })=893 \pm 3 \mathrm{~K},
$$

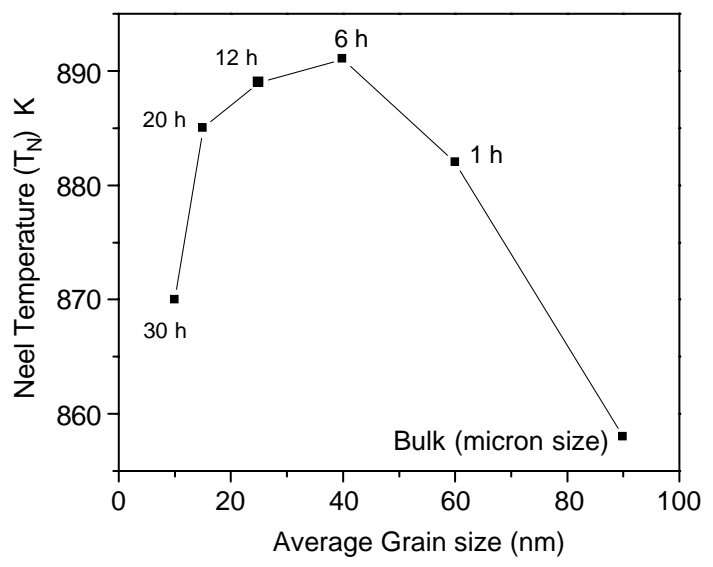

Fig. 4. Néel temperature versus average grain size for nanostructured $\mathrm{NiFe}_{2} \mathrm{O}_{4}$. The continuous line is guide to the eye. 


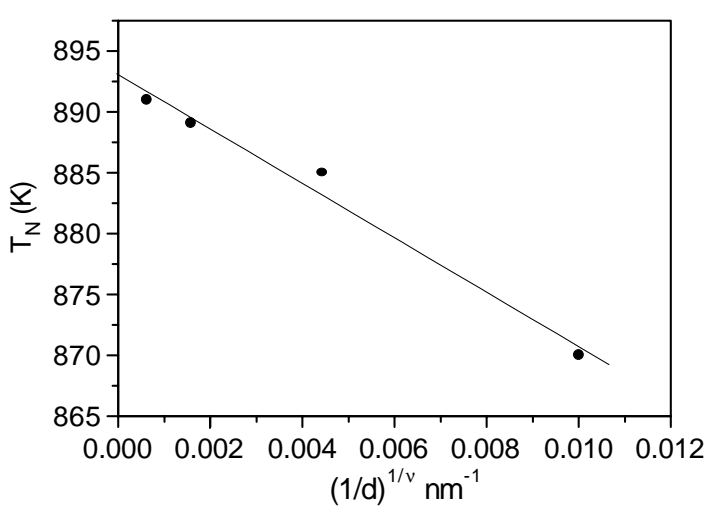

Fig. 5. Finite size scaling analysis of Néel temperature versus grain size. Solid line is obtained by fitting the data for the samples milled for $6,12,20$ and $30 \mathrm{~h}$.

$v=0.5 \pm 0.1$

$d_{0}=1.6 \pm 0.1 \mathrm{~nm}$.

The higher value for $T_{\mathrm{N}}$ (bulk) obtained through the fitting compared to the value of $858 \mathrm{~K}$ obtained for micron size $\mathrm{NiFe}_{2} \mathrm{O}_{4}$ particles [19] indicates that the change in cation distribution in the present samples has increased the net super exchange interaction strength as has been observed in the case of $\mathrm{MnFe}_{2} \mathrm{O}_{4}$ [16]. The variation in Néel temperature of $\mathrm{NiFe}_{2} \mathrm{O}_{4}$ with grain size can be qualitatively explained by combining the effect of change in cation distributions and finite size effects. The increase in the value of $T_{\mathrm{N}}$ for the initial stages of milling ( 1 and $6 \mathrm{~h}$ ) is due to the predominance of the effect due to change in cation distribution over the finite size effect on Néel temperature. We have reported earlier that $\mathrm{Ni}^{2+}$ ions occupy the tetrahedral sites in $\mathrm{NiFe}_{2} \mathrm{O}_{4}$ when ball milled [15]. The exchange constants for the $\mathrm{Ni}-\mathrm{Ni}$ and $\mathrm{Ni}-\mathrm{Fe}$ interactions in the A-sites are not available in the literature to quantitatively account for the variation of Néel temperature with change in cation distribution. The finite size effect should be predominant over the influence of the change in cation distribution when the grain sizes are very small (for prolonged milling time) which results in a decrease in $T_{\mathrm{N}}$ with grain size reduction. The surface effects were dominant for the 20 and $30 \mathrm{~h}$ milled samples as confirmed from our Mössbauer and high field magnetization measurements reported earlier [15]. The small particles have a significant fraction of atoms on the surface and their exchange interaction should be weaker because of the lower coordination and hence they will have a reduced average Néel temperature compared to that of the interior atoms. This will account for the decrease in $T_{\mathrm{N}}$ for higher milling times.

\subsection{Temperature dependence of magnetization and coercivity}

The saturation magnetization and remanence of the $20 \mathrm{~h}$ milled $(15 \mathrm{~nm}) \mathrm{NiFe}_{2} \mathrm{O}_{4}$ sample determined in an applied magnetic field of $1 \mathrm{~T}$ increases on cooling from 300 to $150 \mathrm{~K}$ but on further cooling it decreases both in the case of ZFC and FC states as seen from Fig. 6. This is a clear
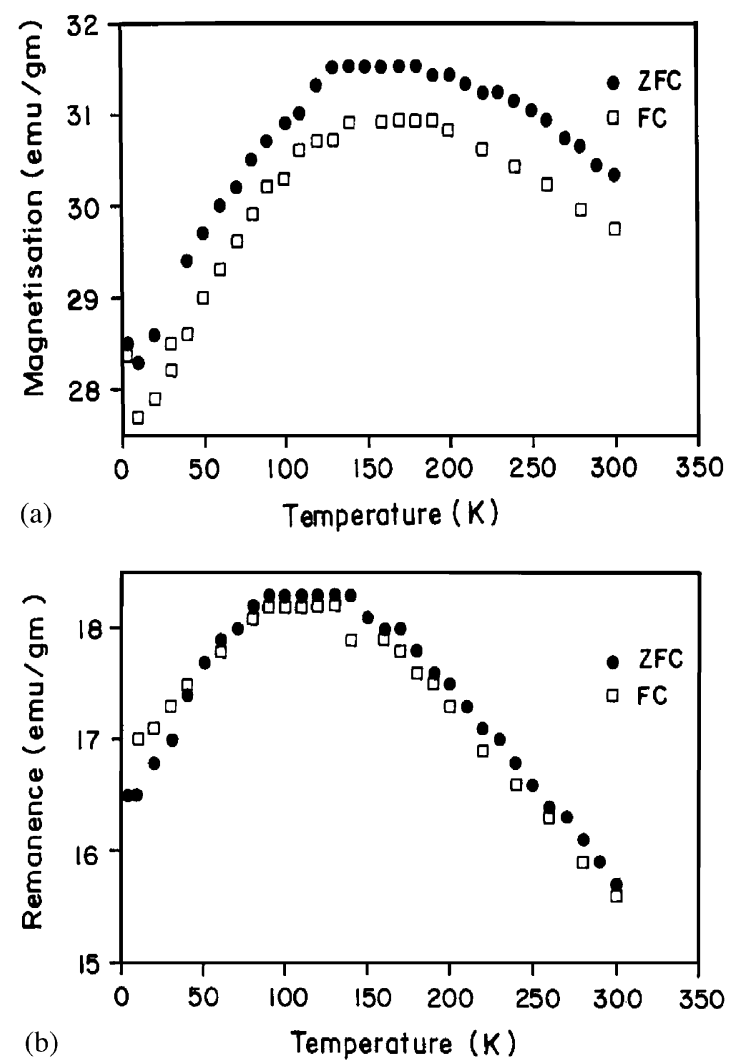

Fig. 6. ZFC and FC curves for the $20 \mathrm{~h}$ milled $(15 \mathrm{~nm}) \mathrm{NiFe}_{2} \mathrm{O}_{4}$ in $1 \mathrm{~T}$ applied field versus temperature (a) for magnetisation and (b) for remanence. 
evidence for the spin canting. A field of $1 \mathrm{~T}$ was found to be small to turn all the spins along the field direction as is evident from the decrease of magnetization below $150 \mathrm{~K}$. The coercivity was found to be $0.22 \mathrm{~T}$ at $5 \mathrm{~K}$ and it decreased to $0.13 \mathrm{~T}$ at $300 \mathrm{~K}$ as seen from Fig. 7. Magnetocrystalline anisotropy, surface anisotropy and shape anisotropy may all contribute to such a high coercivity of ultrafine particles. The hysteresis loop shift was also observed and its value in the FC state for the $1 \mathrm{~T}$ applied field is plotted in Fig. 7 as a function of temperature. The similar temperature dependence of coercivity and the loop shift suggested that the core spins were strongly coupled to the disordered surface spins. The observed loop shift can be understood as explained already by Kodama et al. [14]. Ferrimagnetic ordering in spinel ferrites arises due to the intersublattice $(\mathrm{A}-\mathrm{O}-\mathrm{B})$ super exchange interaction being stronger than the intrasublattice (A-O-A or $\mathrm{B}-\mathrm{O}-\mathrm{B})$ super exchange interactions. Since the surface cations have a reduced and also a distributed coordination, the exchange fields are also distributed. As the super exchange interactions take place through an intervening $\mathrm{O}^{2-}$ ion, exchange bonds will be broken if an $\mathrm{O}^{2-}$ ion is missing from the surface. Also, the surface spins have multiple configurations, i.e. randomly oriented spin directions. Due to the exchange coupling between the surface and core spins, field

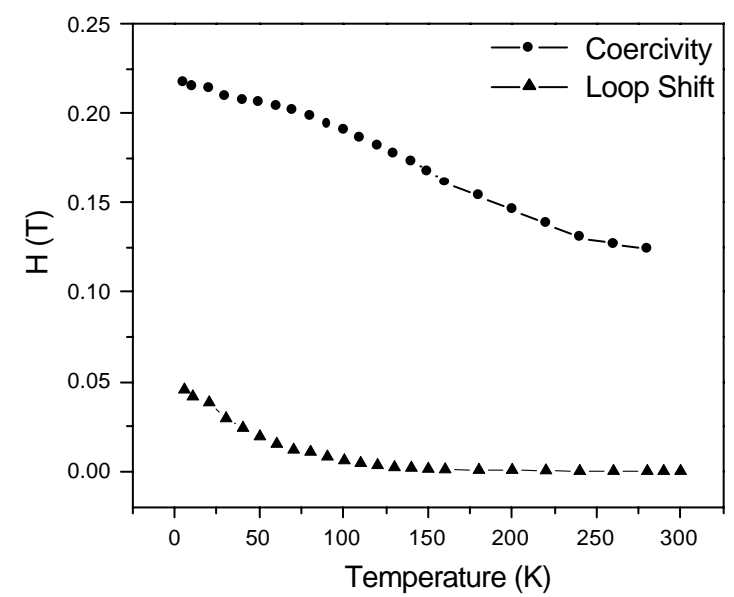

Fig. 7. Coercivity and loop shift versus temperature for the $20 \mathrm{~h}$ milled $(15 \mathrm{~nm}) \mathrm{NiFe}_{2} \mathrm{O}_{4}$ sample in the $\mathrm{FC}$ state. The continuous line is guide to the eye. cooling can select a particular surface spin configuration which favours the particle being magnetized in the FC direction resulting in a shifted hysteresis loop.

\subsection{Hopkinson effect}

The thermomagnetic curves obtained in a magnetic field of $4 \mathrm{mT}$ for the $\mathrm{NiFe}_{2} \mathrm{O}_{4}$ sample with a grain size of $15 \mathrm{~nm}$ are presented in Fig. 8 both for the as-milled sample and for the same sample for a second run. For the virgin sample the magnetization increases with temperature close to the Néel temperature, $T_{\mathrm{N}}$ and have a well-defined maximum just below the Néel temperature. The shape of the curve shows the characteristic peculiarities of the Hopkinson effect [20-22]. However, for the second run the magnetization decreases monotonically with temperature. The increase in the magnetization close to $T_{\mathrm{N}}$ during the first run may, therefore, be due to the Hopkinson effect as has been observed in the case of nanostructured $\mathrm{NiFe}_{2} \mathrm{O}_{4}$ particles synthesized by the citrate precursor technique [23]. Hopkinson effect is observed in nanocrystalline single domain $\mathrm{NiFe}_{2} \mathrm{O}_{4}$ particles where the size of the paticles is

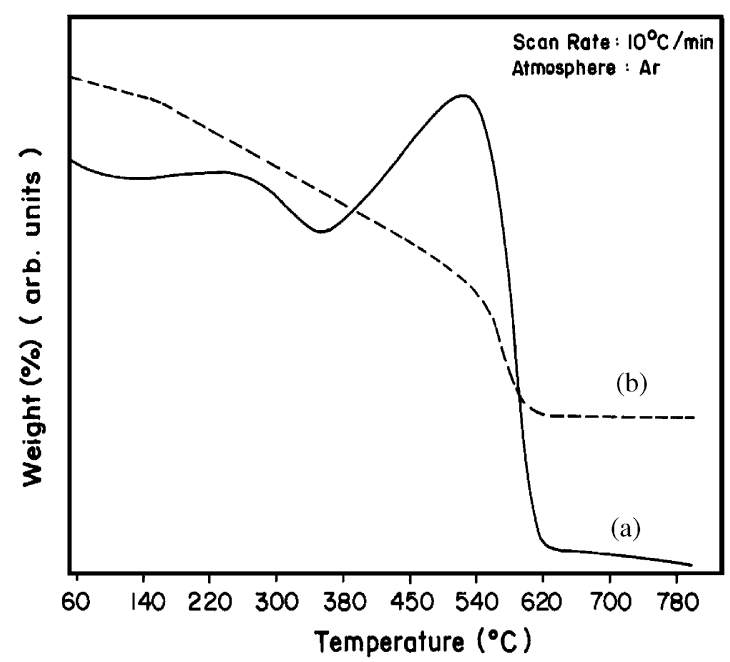

Fig. 8. Thermomagnetic plot for the $20 \mathrm{~h}$ milled $(15 \mathrm{~nm})$ nanostructured $\mathrm{NiFe}_{2} \mathrm{O}_{4}$ : (a) as-milled powder and (b) rerun for the same sample. 
neither very small to be superparamagnetic nor very large to be of multidomain nature [24].

The existence of the Hopkinson effect in the $\mathrm{NiFe}_{2} \mathrm{O}_{4}$ single domain particles can be explained within the framework of the Stoner Wohlfarth model [25]. The magnetization of a randomly oriented system in a small magnetic field, $H$ is

$M_{\mathrm{SW}}(T)=\frac{2}{3} \rho M_{\mathrm{S}}(T) \frac{H}{H_{\mathrm{a}}(T)}$,

where $M_{\mathrm{S}}(T)$ is the bulk saturation magnetization, $H_{\mathrm{a}}(T)$ is the anisotropy field of the particles at the temperature $T$ and $\rho$ is the packing fraction of the powder. When the field $H$ is small, $H_{\mathrm{a}}$ decreases much faster than $M_{\mathrm{S}}$ with temperature and hence there is an overall increase in magnetization which attains a maximum just below $T_{\mathrm{N}}$. Because both $M_{\mathrm{S}}$ and $H_{\mathrm{a}}$ decrease with increasing $T$, this competition may result in a maximum of magnetization, the result is Hopkinson peak. At temperatures far from $T_{\mathrm{N}}, H_{\mathrm{a}}$ is predominant. During the heating run, near $T_{\mathrm{N}}, H_{\mathrm{a}}(T)$ decreasing much faster than $M_{\mathrm{s}}(T)$ and becoming very weak, the magnetic moments of the particles tend to align themselves nearer to $H$ direction. This competitive effect results in a gradual rise of over all magnetization and $M_{\mathrm{SW}}(T)$ shows a steep rise just below $T_{\mathrm{N}}$ since $H_{\mathrm{a}}(T)$ becomes almost zero. Further increase of temperature causes demagnetization and magnetization value approaches to zero at the Néel temperature $T_{\mathrm{N}}$. Since the magnetic moments are already aligned along the field direction due to reduction in $H_{\mathrm{a}}$ during heating in the first run itself, the magnetization decreases monotonically with temperature during the second run.

\section{Conclusion}

The Néel temperatures of the ball milled nanocrystalline $\mathrm{NiFe}_{2} \mathrm{O}_{4}$ spinel ferrites are found to be greater than that of the bulk $\mathrm{NiFe}_{2} \mathrm{O}_{4}$. The variation of the Néel temperature with grain size has been explained on the basis of finite size effects and changes in cation distributions. The observed hysteresis loop shift has been attributed to the canting of surface spins and possible multiple surface spin configurations. The Hopkinson effect was explained within the framework of the mathematical formalism given by Stoner Wohlfarth.

\section{Acknowledgements}

The financial assistance from UGC-SAP (PhaseIII) and UGC Major Research Project (F.10-32/ 98(SR-I)) sanctioned to the University of Madras is gratefully acknowledged. The authors would like to thank Dr. R. Krishnan for fruitful discussions. Two of us (CNC and NP) would like to thank the Council of Scientific and Industrial Research, Government of India for the award of senior research fellowship.

\section{References}

[1] W. Schissel, W. Potzel, H. Karzel, M. Steiner, G.M. Kalvius, A. Martin, M.K. Karause, I. Halevy, J. Gal, W. Schäfer, R. Wäppling, Phys. Rev. B 53 (1996) 9143.

[2] H.H. Hamdeh, J.C. Ho, S.A. Oliver, R.J. Wiley, G. Oliveri, G. Busca, J. Appl. Phys. 81 (1997) 1851.

[3] M. Clark Ted, B.J. Evans, IEEE Trans. Magn. 33 (1997) 3745.

[4] M. Yokayama, E. Ohta, T. Sato, T. Komaba, T. Sato, J. Phys. Coll. IV 7C1 (1997) 521.

[5] J. Battle, T. Clark, B.J. Evans, J. Phys. Coll. IV 7C1 (1997) 257.

[6] H.H. Hamdeh, J.C. Ho, S.A. Oliver, R.J. Wiley, J. Kramer, Y.Y. Chen, S.H. Lin, Y.D. Yao, M. Datiro, G. Busca, IEEE Trans. Magn. 31 (1995) 3808.

[7] T. Sato, K. Haneda, M. Seki, T. Iijima, Appl. Phys. A 50 (1990) 13.

[8] M.R. Anantharaman, S. Jagatheesan, K.A. Malini, S. Sindhu, A. Narayanasamy, C.N. Chinnasamy, J.P. Jacips, S. Reijne, H. Seshan, R.H.H. Brongersma, J. Magn. Magn. Mater. 189 (1998) 83.

[9] F.J. Burghart, W. Potzel, G.M. Kalvius, E. Schreier, G. Grosse, D.R. Noakes, W. Schäfer, W. Kockelmann, S.J. Campbell, W.A. Kaczmarek, A. Martin, M.K. Krause, Physica B 289-290 (2000) 286.

[10] C.N. Chinnasamy, A. Narayansamy, N. Ponpandian, K. Chattopadhyay, H. Guérault, J.-M. Greneche, J. Phys.: Condens. Matter 12 (2000) 7795.

[11] C.N. Chinnasamy, A. Narayanasamy, N. Ponpandian, R. Justin Joseyphus, K. Chattopadhyay, K. Shinoda, B. Jeyadevan, K. Tohji, K. Nakatsuka, J.-M. Greneche, J. Appl. Phys. 90 (2001) 527. 
[12] B. Jeyadevan, K. Tohji, K. Nakatsuka, J. Appl. Phys. 76 (1994) 6325.

[13] A.H. Morrish, K. Haneda, J. Appl. Phys. 52 (1981) 2496.

[14] R.H. Kodama, A.E. Berkowitz, Phys. Rev. B 59 (1999) 6321.

[15] C.N. Chinnasamy, A. Narayanasamy, N. Ponpandian, K. Chattopadhyay, K. Shinoda, B. Jeyadevan, K. Tohji, K. Nakatsuka, T. Furubayashi, I. Nakatani, Phys. Rev. B 63 (2001) 184108.

[16] J.P. Chen, C.M. Sorenson, K.J. Klabunde, G.C. Hadjipanayis, E. Devilin, A. Kostikas, Phys. Rev. B 54 (1996) 9288.

[17] D. Olezak, Mater. Sci. Eng. A 181/182 (1994) 121.

[18] T. Sato, T. Iijima, M. Seki, N. Inagaki, J. Magn. Magn. Mater. 65 (1987) 252.
[19] J. Smith, H.P.J. Wijn, Ferrites, Philips Technical Library, Eindhoven, The Netherlands, 1959, p. 157.

[20] J. Hopkinson, Proc. R. Soc. London 48 (1890) 1.

[21] O. Popov, P. Rachev, M. Mikhov, F. Calderon, J.L. Sanchez, F. Leccabue, J. Magn. Magn. Mater. 99 (1991) 119.

[22] O. Popov, M. Mikhov, J. Magn. Magn. Mater. 82 (1989) 29.

[23] S. Prasad, N.S. Gajbhioe, J. Alloys Compds. 265 (1998) 87.

[24] N.S. Gajbhiye, S. Prasad, G. Balaji, IEEE Trans. Magn. 35 (1999) 2155.

[25] E.C. Stoner, E.P. Wohlfarth, Phil. Trans. Roy. Soc. A 240 (1948) 599. 